\title{
Interdisciplinary Technologies in Socio-Political Rehabilitation
}

\author{
Oleksandr Malkhazov ${ }^{1}$, Sergiy Osypenko ${ }^{2}$ \\ ${ }^{1}$ Institute of Social and Political Psychology, National Academy of Educational Sciences of \\ Ukraine, Kyiv, Ukraine \\ ${ }^{2}$ G. S. Kostiuk Institute of Psychology, National Academy of Educational Sciences of \\ Ukraine, Kyiv, Ukraine
}

Introduction. One of the ways to improve psychological support of the rehabilitation measures in the field of psychological health is to apply technological resources from different areas of psychological knowledge. This means first considering psychophysiological indicators and individual characteristics of the people who need help at different stages of socio-psychological rehabilitation in order to carry out a complex diagnostics of the factors of psychological illness and means of addressing them. The obstacles in the way are, on one hand, lack of interdisciplinary technologies aimed at solving psychological health problems, and, on the other, lack of diagnostic equipment.

Purpose. The purpose of the research is to develop a technology of socio-political rehabilitation for individuals who experienced a traumatic event or suffer from PTSD. The technology is supposed to use a modern diagnostic device system to measure psychological and psychophysiological characteristics of an individual.

The research allowed verification of the assumption that step by step control of the dynamics of the changes occurring in the state of the patient under the influence of the rehabilitation measures increases effectiveness of the psychological support.

To develop the ability to regulate the emotional states of individuals who have experienced a posttraumatic event it is suggested to apply the technology of socio-psychological rehabilitation, which can be implemented with the help of special equipment. Therefore, one of the tasks of the study was to develop a diagnostic system to perform step-by-step control of effectiveness of the rehabilitation procedures.

Methodology. The first preparatory stage envisaged developing the technology for increasing the emotional resilience of the individuals experiencing consequences of traumatic events on the basis of analyzing their current problems and technological approaches to emotional state regulation.

Our next step (methodological stage of the study) focused on selecting the methods relevant to the tasks perused at each stage of the developed technology.

To reach the established objectives the following groups of methods were used:

methods aimed at determining the degree of the patient's psychophysiological and emotional exhaustion, optimal exertion and recuperation mode, as well as duration of the testing and training sessions (chronometry and methods of registering neurodynamic characteristics: tapping test, reaction to moving objects, accuracy of time estimation, critical flicker frequency); 
methods aimed at determining the level of emotional stress (galvanic skin response, photoplethysm, oculography);

methods aimed at identifying the patient's individual characteristics (extra- and introversion, neurotism, impulsivity, excitedness, communicability, reflexivity, anxiety, demonstrativeness, stuckness, hypertensiveness, dysthymia, cyclotimity, exaltation, emotivity, steadiness, and social maturity). In order to diagnose the above mentioned qualities the questionnaires by Eysenck, Leonhard, Thurstone, Strelau, Leary and Rosenzweig were used.

The third instrumental stage of the study was devoted to the development and piloting of a sample diagnostic system. The piloting stage envisaged training based on the biofeedback principle. The biofeedback method is used to change respondents' attitudes to themselves by enhancing their belief in their own capability to control their emotional states. Emotion control skills on the psychophysiological level are further transformed into the models of emotional state control in different situations of social interaction. To this end, situation modeling methods, reflective analysis and projective methods are applied.

Results. Our approach to the development of the system is based on the ideas of W. Boucsein (2012) and interactive psychophysiology (İalkhazov, 2014), which were applied in the course of the diagnostic system development (İalkhazov, 2008). We took into consideration the experience of creation and application of similar devices and techniques by Duric-Jovicic, M., Jovicic, N., Radovanovic, S., Jecmenica-Lukic, M., Belic, M., Popovic, M., \& Kostic, V. (2018), Hagger M. S., Luszczynska, A., de Wit, J. et al (2016), Schwartz, M. S., \& Andrasik, F. (Eds.). (2017), Wahbeh, H., Goodrich, E., Goy, E. Et al (2016).

Unlike the works mentioned above the proposed device system is multifunctional. It can be used to solve a wide range of psychic health problems, particularly those occurring in the process of socio-psychological, psychophysiological and medical rehabilitation, and when making and testing bio prosthesis, bio robots, trainers, etc. The device system makes it possible to register with high accuracy quantity indicators of psychophysiological and individual peculiarities of a respondent in real time. Thanks to the original software and mathematical provision, the current and final results of the test are immediately displayed on the researcher's monitor. On the basis of the diagnostic data obtained, a choice of rehabilitation strategies is made.

The device system consists of two items, which can function both in the autonomous and multifunctional modes, a computer provided with the original software, and two monitors: one is for the respondent and the other for the rehabilitation specialist.

The first device, which serves to diagnose neurodynamic and chronometric parameters, is used at the first stage and partly before the second and the third implementation stages of the technology developed. The device helps to display information essential for determination and control of the exertion and recuperation modes, which is drawn from the analysis of the investigation results on the monitor of the rehabilitation specialist.

The second device, which serves to register emotional exhaustion parameters, enables the rehabilitation specialist to follow changes in emotion and emotional states of the respondent, degree of increase or decrease of his/her emotional exhaustion when reacting to specific 
questions, statements, words, etc. That ensures substantially higher screening reliability. The information obtained is taken into account on the reflective and partly on the formation stages of the technology implementation.

The advantages of the interdisciplinary approach can be demonstrated using the example of the technology of enhancing emotional resilience of the individuals experiencing consequences of traumatic events.

In the framework of this study, emotional resilience is defined as the ability of an individual to control his/her emotional states in situations of significant of social interaction and in his/her professional activities. This ability is the result of the functioning of a multilevel system of psychophysiological, individual psychological and socio-psychological factors. It may be assumed that the system effectiveness can be changed by correcting its single elements.

It is common knowledge that emotional resilience of individuals experiencing consequences of traumatic events, to a greater extent, depend on their own ability to control their emotions and emotional states in the course of social interaction. Therefore, it is the development of the individual capacity for self-control that is seen as a resource for enhancing emotional resilience.

The proposed technology is implemented in three stages. At every rehabilitation stage, the diagnostic device system is used. It includes two monitors, one of which is placed in front of the respondent and the other is in the researcher's field of view. The respondent's unit is equipped with three video cameras, which record his/her eye motions. Besides, there are photoplethysm and galvanic skin response sensors on the respondent's fingers.

During the first diagnostic stage, a respondent following the researcher's request speaks about him-/herself, remembers the situations when he/she experienced strong emotions (fear, happiness, joy, rage, anger, annoyance, etc), describes the cases when he/she managed to gain control over his/her emotions, analyzes concrete emotionally significant situations of his/her past, and tries to model different variants of their desired solution. The next step is the identification of the individual psychological profile of the respondent by means of questionnaires, with simultaneous registration of the psychophysiological indicators; this allows revealing the truthfulness of the statements and the semantic statement clusters that cause tension.

The results of the diagnostics in real time provide the researcher with information that helps to draw conclusions about the ability of the individual to differentiate his/her own emotions and emotional states, his/her experience and the level of reflection on his/her own emotional manifestations, as well as the ability to distinguish the situations that lead to a decrease in emotional resilience. The researcher identifies typical situations in which the respondent loses the ability to control his/her emotions and to apply most frequently used coping strategies (experience of avoidance, experience of successful solution, and other ways of coping).

During the second reflexive stage the psychotherapist specifies his/her understanding of the individual peculiarities, resources and self-regulation problem zones of the respondent by determining and analyzing the conditions and factors of his/her emotional reactions; modeling successful and unsuccessful ways of coping with the emotional states with further analysis of 
the reasons for failure; applying technological techniques to enhance the respondent's belief in his/her own ability to regulate his/her emotions.

With a view to the objective above, the psychotherapist suggests that the respondent analyze the situation in which he/she regularly lacks emotional resilience and determine its causes (internal and external ones). The researcher and the respondent discuss possible ways of overcoming the internal and external causes and precisely formulate those that are in the zone of the respondent's potential control. They work out the repertoire of the ways to cope with the emotions that is already used by the respondent, as well as the ways which he/she could use. In order to prove the respondent's ability to regulate his/her own emotions, biofeedback learning techniques can be applied.

The aim of the third formation stage is to specify the idea of the desired models of the respondent's behavior in the emotionally significant situations and to determine the necessary abilities and skills for emotion correction. At this stage, rehabilitation techniques of enhancing confidence in one's ability to control emotional states are applied. The respondent masters the techniques of emotion regulation in the typical situations and develops the ability to anticipate and choose the optimal model of behavior in the situations causing the decrease of emotional resilience (avoidance, solution, changing attitude about the situation, suppression or depreciation of the situations that cannot be solved).

The diagnostic system is used on the third stage to master respondents' skills to distinguish emotions, identify situations that cause emotional reactions, independently regulate emotional states and learn some techniques to preserve self-control. In the process of rehabilitation, the control of the emotional state practices acquired through applying the biofeedback method are transformed into the models of behavior in the potentially provocative situations of social interaction.

Thus, the application of the psychodiagnostic device system makes it possible to receive information about the individual psychophysiological and psychological peculiarities of a patient in real time. On the basis of the data obtained, the psychoanalyst determines the strategy of his work, chooses optimal load regimes, identifies compensation possibilities and controls the effectiveness of the rehabilitation measures.

Keywords: interdisciplinary approach, emotional resilience, rehabilitation, technology, psychodiagnostic device system.

\section{References.}

Boucsein, W. (2012). Electrodermal activity. Springer Science \& Business Media.

Duric-Jovicic, M., Jovicic, N., Radovanovic, S., Jecmenica-Lukic, M., Belic, M., Popovic, M., \& Kostic, V. (2018). Finger and foot tapping sensor system for objective motor assessment. Vojnosanitetski pregled, 75(1), 68-77. 
Hagger, M. S., Luszczynska, A., de Wit, J., Benyamini, Y., Burkert, S., Chamberland, P. E., \& Gauchet, A. (2016). Implementation intention and planning interventions in Health Psychology: Recommendations from the Synergy Expert Group for research and practice. Psychology \& health, 31(7), 814-839.

İàlkhazov, Î.R. (2008). New solutions to the problem of the psychological support of an operator's safety. Proceedings of the Second World Congress "Aviation in the XXI - st century" "Safety in aviation and space technology". Vol.2. - K.: NAU., 6.286.32 .

İàlkhazov, Î.R. (2014) Integrative psychophysiology: methodological foundations. In ${ }^{222}$ AllUkrainian Psychological Congress with International participant «Personality in the modern world». E..: DP «Information and analytical agency», 53 - 57.

Schwartz, M. S., \& Andrasik, F. (Eds.). (2017). Biofeedback: A practitioner's guide. Guilford Publications.

Wahbeh, H., Goodrich, E., Goy, E., \& Oken, B. S. (2016). Mechanistic pathways of mindfulness meditation in combat veterans with posttraumatic stress disorder. Journal of clinical psychology, 72(4), 365-383. 